\title{
Review
}

\section{DIAGNOSTICS OF INTRAPULMONARY METASTASES FROM COLORECTAL CANCER (LITERATURE SURVEY)}

\author{
E. Obretenov ${ }^{*}$, N. Georgieva ${ }^{2}$ \\ ${ }^{1}$ Clinic of Thoracic Surgery UMBAL „Prof. d-r S. Kirkovich”, Stara Zagora, Bulgaria \\ ${ }^{2}$ Department of Physics, Biophysics, Roentgenology and Radiology, Medical Faculty, \\ Trakia University, Stara Zagora, Bulgaria
}

\begin{abstract}
The colon cancer is on the first place as etiologic cause for performing pulmonary resection due to pulmonary metastases. The low effectiveness of chemotherapy in case of metastatic progression from colorectal carcinoma is established. Accordingly, the surgical resection is commonly accepted as the only potential therapeutic option. In favour of the good results from surgical treatment is the trend of earlier finding of pulmonary metastases. The metastases diagnostics has important meaning for differentiation from other lung diseases and for the choice of the therapeutic method. The benefits and disadvantages of non-invasive (chest X-ray, CT, lung perfusion scintigraphy, PET and PET/CT, tumor markers) and invasive (bronchoscopy, mediastinoscopy, VATS (video-assisted thoracic surgery, TTNB (trans-thoracic needle biopsy)) methods for diagnosis of intrapulmonary metastases are analyzed.
\end{abstract}

Key words: colorectal cancer, pulmonary metastases

There are different reports in the scientific literature about the incidence of colorectal carcinoma. According to some authors it is the third most often diagnosed cancer in the world (1-4). The data from P. J Villeneuve and R. S. Sundaresan (5) show that over one million new patients get sick from this insidious disease every year. H. Meulendeld at al. (6) report that $40 \%$ of the patients with colorectal carcinoma in Europe are over 75 years of age at the moment of first diagnosis and there are expectations that this number would significantly increase in the next two decades.

The colorectal carcinoma in Bulgaria according to the data from 2012 for prevalence is on third place in men and on second place in women and represents $8.6 \%$ from all malignant diseases totally for both sexes. The registered new cases are 2772 , and $52,7 \%$ of them in men. The diagnosis in $42,4 \%$ of the patients is set in advanced III and IV stages. The relative 5 - year survival in Bulgaria is $45,0 \%$ for men, $45,3 \%$ for women and $45,2 \%$ totally for both sexes. It is lower than the average for Europe$55,6 \%$. (7). According to summed statistics A.

\footnotetext{
*Correspondence to: Evelin Obretenov, Clinic of Thoracic Surgery, University Hospital Stara Zagora, 2 , Gen. Stoletov” str. 6000 Stara Zagora,Bulgaria,evelin.obretenov@gmail.com
}

Sach, S. Alberts and R. Adam (8) report that colon carcinoma is the fourth most distributed cancer and it is second most frequent cause for mortality of the patients with oncologic diseases. Approximately $35 \%$ from the diagnosed patients are in stage IV and $20 \%$ to $50 \%$ of the patients in stage II and III reach stage IV despite the treatment (8).

The frequency of the isolated pulmonary metastases is in the range $1,7 \%$ to $11,4 \%$ (914). This is explained by the fact that the venous drainage of medium and lower third of the rectum is through lower and medium rectal veins, avoiding the venous system of v.portae and respectively, the liver which is the first filter $(13,15,16)$. N. Rama at al. (17) registered that the lung metastases predominate when the localization is in lower and medium third of the rectum, in $36 \%$ and $39 \%$ respectively versus the rest $25 \%$ of the patients with localization in the upper third of the rectum. W. Takahashi et al. (1) report that after total removal and treatment of the patients with colorectal carcinoma pulmonary progression is registered approximately in $10 \%$.

The diagnosis is often set according to the clinical symptoms, but usually the pulmonary metastases are asymptomatic (18-20). Clinically they stay undeveloped for long time and barely express in the advanced stages. The 
early establishment of the pulmonary progression is of significant importance for the treatment outcome.

\section{DIAGNOSTICS}

S. Jegatheeswaran et al. (21) report that almost in all patients (99\%) with colorectal carcinoma, to exclude metastatic progression or relapse a chest computer tomography (CT) is performed; in $70 \%$ of the patients a CT of the liver is needed and additionally $\mathrm{CT}$ of the pelvis in $51 \%$ of the patients is applied. Positron emission tomography (PET) is used in $86 \%$ of the diseased (21).

The diagnostic process of pulmonary metastases from colorectal carcinoma includes several main stages:

Chest X-ray - C. Gielen et al. (22) claim that the X-ray of the lungs has low percentage of finding of the pulmonary metastases and insignificant influence on the treatment of the patient. Their data indicate $0,86 \%$ finding ability of the pulmonary neoplastic lesions with confidence interval 0,3-1,4 \%. In accordance with numerous examinations, the authors do not support the routine usage of the conventional X-ray of the thorax in patients with colorectal cancer (22). Other publications report that this method for establishing of pulmonary metastases is effective in $33 \%$ to $40 \%$ of the cases $(10,23,24)$. Based on a literature analysis of the problem I.Grossmann et al. (10) establish that by X-ray the pulmonary metastases could be visualized in up to $55 \%$ of the patients. Similar are the data of J. H. Lee et al. (23), who report based on summed statistics that in $67 \%$ of the cases there is benefit of the conventional X-ray for diagnosis of the pulmonary metastatic progression in patients with colorectal cancer.

The early X-rays register formation of network drawing, over which are projected separate small-spotted shadows - the future nodes. The most often X-ray findings according to the different authors are solitary or multiple nodes in consolidation $(23,25,26)$. The X-ray image is characterized with differently large round or oval shadow, with significant X-ray intensity, uniform structure, with sharp and smooth, sometimes polycyclic outlines. Rarely a cavity lightening in the tumor core is found. The endobronchial development of the tumor is shown by findings of atelectasis area. The advance of the tumor process is characterized by bronchia compression, expansion in neighbouring lobes and germination in the pleura and the chest wall. The X-ray image often resembles focal pneumonia and is characterized with the presence of thick spotted shadows, showing a tendency for quick growing and confluence. According to the data from J.H. Kim et al. (25) the X-ray in endobronchial metastases shows obstructive atelectasis and diffuse infiltration of pulmonary parenchyma. The signs of obturative pneumonia are frequent. C. Fournel et al. (26) report that the most frequent X-ray finding in endobronchial metastases is atelectasis in $58 \%$ of the cases, followed by pulmonary node in $25 \%$, pulmonary infiltration in $8,3 \%$, pleural collections in $4,2 \%$, hilar formations in $12,5 \%$ and mediastinal limphadenomegaly in $8,3 \%$ of patients.

The differential diagnosis of tumor metastases by X-ray is complex. In isolated lesions the benignant tumors come into consideration, echinococcosis cyst, tuberculoma and in multiple metastases - disseminated tuberculosis, sarcoidosis, pneumoconiosis, focal pneumonia and etc. Decisive for the diagnosis is the detection and establishment of the primary tumor process.

Computed tomography of the thorax - The computed tomography of the chest (CT) has become an important additional tool for the doctor, which is usually used for finding of a tumor disease, evaluation of the therapeutic response and the appearance of relapse (27). Performing a literature analysis about the effectiveness in diagnosis of pulmonary metastases through conventional X-ray and CT of the thorax M.W. Christoffersen et al. (24) report a low sensitivity of the first around 33\%, in comparison with $\mathrm{CT}$ which reaches sensitivity of $73 \%$. In the recent years computed tomography has become a main method for finding pulmonary metastases (1, 28-33). A. Nordholm-Carstensen et al. (28) advise its performing as a part of the routine care for the oncology patient in the first two years, because the analyses indicate that $84 \%$ of the malignant pulmonary lesions develop in this period. (28). T. Kawaguchi et al. (34) describe $\mathrm{CT}$ changes in pulmonary metastases like tumor formations with smooth and sharp borders in $42 \%$ or formations with indistinct borders and shape in 56\% of the patients. Registered frequently are also spiculative growth aside with pulling of the pleura. Cavity shadows could be observed in them, entirely empty or partially filled with necrotic materia in $26 \%$ and existing calcification in $18 \%$ of the cases. Except of the pulmonary finding CT could be useful for evaluation of the hilar and mediastinal lymph nodes. Size over $1,0 \mathrm{~cm}$ is determined as positive for lymphadenopathy (15). 
In spite of all advantages of CT we should note that it has lower specificity for registered undefined lesions of the lung. Their origin remains a serious problem, because they are a frequent finding in $20 \%$ to $30 \%$ of the cases. According to some of the authors these are malignant in 10\%-20\% (10), but according to other publications the detected node in the lungs in $65 \%$ is a metastasis, and in the rest $35 \%$ - benignant lesion (35). According to Y. Varol et al. (27) as a suspicious, undefined pulmonary node is defined one or more formations in the pulmonary parenchyma with size less than $1,5 \mathrm{~cm}$ in diameter.

P. Jess et al. (32) report that by literature data 4 to $42 \%$ of the patients with colorectal carcinoma have pulmonary formations found by CT. In their study this percentage has been $20 \%$. In $8,5 \%$ they turned to be pulmonary metastases, diagnosed 9 months after operation of colorectal carcinoma. In 4,3\% has been established that another neoplastic disease is concerned, in $2,1 \%$ it is tuberculosis and in $5,4 \%$ for pulmonary metastases developed 16 months after the operation for colorectal cancer and normal CT scan before the operation. Despite that the number of unidentified pulmonary lesions which turn to be malignant is relatively small, it is recommended to use $\mathrm{PET/CT}$ scan in the subsequent actions for finding the pulmonary metastases at earliest possible in order to improve the prognosis. The ascertained results oblige us to look hard the histologic verification of the undefined lesions. By the same reason all of the patients with colorectal carcinoma should be examined by CT of the thorax in the following 6-12 months after the operation $(32,36)$. The metastases appear at average 2-3 years after removal of the primary tumor (18). Based on the data from A. Nordholm-Carstensen et al. (37), after the routine examination with pre-operative CT of the thorax in $9 \%$ undefined pulmonary nodes have been found. From them $10,8 \%$ turned to be metastases from colorectal cancer and because of this fact a conclusion is driven that only 1 from 100 patients (1\%) with colorectal carcinoma undergoing pre-operative scan would have undefined pulmonary lesion which could be detected as metastasis from the main disease. The subsequent low risk suggests that unclear pulmonary finding should not cause additional changes in pre-operative diagnostics and routine schemes of therapeutic management.

In $65,5 \%$ of the patients the lesions are localized peripherally and described as subpleural. In the rest $34,5 \%$, the findings have been disseminated in the lungs and are recognized as parenchymal. In $16,4 \%$ of the patients calcifications in the nodes are found. The characteristics of the lesions belong to two categories - with round $(84 \%)$ and irregular (16\%) form. During the follow-up 31\% of the patients have had progression. Metastases are established in $47 \%$ of them (27). In $35 \%$ of the patients has been determined that metastases' process has occurred during the follow-up period. Half of these metastases have been demonstrated by increased size or number and the other half has been established histologically. In most of the patients with rectal carcinoma the registered nodes are over $1 \mathrm{~cm}$. This fact is explained by the way of vascularization of the rectum (27). The smooth borders and sub-pleural localization are more frequently related to benignant lesions (27). Based on his study Y. Varol et al. (27) come to the conclusion that undefined multiple pulmonary nodes with irregular form and border, localized in parenchyma are more probable to be a metastatic disease. A. Brent et al. (38) report that $10 \%$ of the patients have had undefined changes in the lungs. Half of them have had dissemination in the regional lymph nodes (N1 and N2) at the time of removal of the primary tumor (38).

J. Nakajima et al. (39) recommend realization of spiral CT of the thorax with thickness of the slice $5 \mathrm{~mm}$ contrasted before the eventual pulmonary metastase-ectomy operation. The number, size and localization of pulmonary metastases are followed-up. Pulmonary solid node, sometimes with cavity in it, with $5 \mathrm{~mm}$ diameter or more, without calcification or occurrence of new nodes, even if their diameter is below $5 \mathrm{~mm}$ suggest that these are pulmonary metastases. Patients have been controlled on every 3-6 months by CT and test for carcinoembryonic antigen (CEA) until the finding of relapse of the colorectal carcinoma $(24,39)$.

Patients with colorectal cancer presenting with four or more undefined pulmonary nodes in pre-operative staging by CT, even with lack of metastases in another location, have a big probability for pulmonary progression of the disease (40). The final diagnosis of the pulmonary formations is based on the images from CT (characteristics and growth rate), PET/CT scanning or the histologic verification. The undefined pulmonary lesions are accepted as benign, when there are no signs registered for malignant growth on the second chest scanning (CT) and lacks increase of CEA at least for a year during the follow-up (10). The lesions most often become evidently malignant in the beginning of the second year after the operation (24). 
The small nodes are often seen at CT of the thorax and they usually are benign, The difficulty with these lesions is that they are usually too small (between 0,5 and $1 \mathrm{~cm}$ ) for assessment of their density and their form. The assessment of the nature of these changes is very difficult. PET has the same disadvantage for small formations. Percutaneous needle biopsy could be technically difficult and predisposes towards diagnostic mistakes. When the histologic result does not show malignancy, the metastases could not be excluded with certainty. Complications, like implantation and dissemination of tumor cells, pneumothorax and hemorrhage could be present after this invasive procedure (10). We share the standpoint of other authors that small lesions on CT $(<1 \mathrm{~cm})$ should be regarded with limited clinical meaning and to be neglected in the choice of initial treatment (10). D. Gomez et al. (41) report based on summed statistics that unidentified pulmonary nodes are found in 25\% (varying from 11 to $35 \%$ ) from all patients with colorectal carcinoma after CT of the thorax (10). These patients represent a diagnostic problem. The solution is PET/CT or histologic verification by TTNB (transthoracic needle biopsy) or VATS (videoassisted thoracic surgery) if they are localized peripherally. It should be considered that by PET could not be succeeded to distinguish the metastatic disease from benign inflammatory process, because of the small size of the node (41). As a whole in $50 \%$ of the patients with unidentified pulmonary nodes a metastatic pulmonary disease is found after a mean period of 10 months (41). Despite of all, it is reported that with conventional computer tomographs $50 \%$ of the metastatic pulmonary nodes are missed and they are found during the surgical intervention. In a prospective randomized study Collie et al. establish that this mistake is reduced 2 times by spiral scanning - up to $25 \%$ more lesions are found and confirmed during the surgical resection (42).

C. Marron Fernandez et al. (43) report that there is discrepancy between the diagnosed by $\mathrm{CT}$ and surgically found and resected pulmonary metastases in $22 \%$ of the patients. Their analysis has shown that the discrepancy is more probable in patients with advanced (III, IV) stage of colorectal carcinoma, in nodes with smaller mean size, in diagnosed hepatic metastases, in bilateral (left and right) involvement and in existing multiple nodes on CT (43). Comparing the found metastatic nodes during the $\mathrm{CT}$ with these resected during operation, it is established accuracy of the methodology in $85,3 \%$ of the patients. In cases with single metastasis it has been $89.8 \%$ and in cases with multiple metastasis $-65,8 \%$ (33).
Based on the results cited by J. Zabaleta et al. (33), in the international register of pulmonary metastases, the accuracy of CT in terms of finding the exact number of the pulmonary nodes is $61 \%$. Around $30-40 \%$ of the additionally imaged lesions with CT and resected during the operative treatment have been benign (33). The sensitivity of CT in patients with single metastasis of colorectal carcinoma has been 95\% (33). The pace of discovery of pulmonary metastases by CT with high resolution is $78-84 \%(24,44)$.

P. J. Villeneuve and R. S. Sundersan (5) recommend CT of the thorax with high resolution as a choice and test of first line for diagnostics of the pulmonary lesions, especially after contrasting. This technology gives opportunity for acquiring image of the whole chest with $1 \mathrm{~mm}$ thickness of the slice during one of the phases of inspiration or expiration. The older generation of apparatus are $100 \%$ sensitive for nodes $>6 \mathrm{~mm}$. For the smaller parenchymal lesions the exact sensitivity is achieved in $66 \%$ of the cases. The assessment of the pleural nodes is rather more difficult, as accuracy is achieved only in $17 \%$ for findings with diameter lower or equal to $6 \mathrm{~mm}$. The increased sensitivity to $98 \%$ could be achieved with the help of venous contrast.

PET is a method of choice for discovering occult metastases (patients with increased level of CEA, in whom there is no evidence for the disease), in unsuspected pulmonary or hilar lymph-adenomegaly and for assessment of extra-thoracic burden of the disease (5). In some cases the authors recommend preoperative diagnosis of the pulmonary nodes with TTNB, because this facilitates the operative planning (5). The positive immunehistochemical coloring for TTF1 proves the origin from the lungs or the thyroid gland (91\% sensitive, 98\% specific), and CDX2 coloring excludes the colorectal origin (83\% sensitive, $96 \%$ specific). This differentiation is from essential importance, because the treatment, the setting and the volume of the surgical resection of the primary cancer of the lung differs significantly from this for metastases of colorectal carcinoma. It should be taken into consideration that this method is hindered by the small size of the undefined node and may obtain falsely negative results (5).

R. Schneider et al. (13) report that after spiral CT in around $14 \%$ of the cases metastases are missed, and after surgery intervention they are confirmed. Approximately $44 \%$ of these metastases are less or equal to $2 \mathrm{~mm}, 30 \%$ are less or equal to $4 \mathrm{~mm}$ and $26 \%$ are bigger than 
$4 \mathrm{~mm}$. The overall sensitivity of the spiral CT is $86 \%$ (13). Based on the data of J. Nakajima et al. (39), more than $40 \%$ of the pulmonary nodes with dimensions equal or lower than 5 $\mathrm{mm}$ in diameter, which are found by CT with suspicion of pulmonary metastases, are not confirmed from the histology test.

Lung perfusion scintigraphy - The nuclear medicine examination with ${ }^{99 \mathrm{~m}} \mathrm{Tc}$ - MAA. Solitary metastases of the lungs induce predominantly rounded area of impaired perfusion. In our examinations the single metastases in the lung provoke perfusion alterations which are observed in peripheral lung tumor. When the metastasis is situated in the hilum, a perfusion defect in the whole lobe may be observed. In single solitary metastases in the right lung and in bilateral metastases the perfusion indices are most often around the normal ratios right - left. The involvement of one, or both hili and distent mediastinum are indirect sign for advanced metastatic process or presence of multiple metastases. The metastases in the left lung more often induce larger changes in perfusion indices (45). The examination is almost completely displaced by the perfusion CT and PET/CT.

Positron-emission tomography (PET) and PET/CT - Positron-emission tomography (PET) is a radionuclide method, which is used for non-invasive finding and quantitative determination of cellular and biochemical processes (46). This is a method for functional imaging, which could be combined with CT or MRI and by this way to improve the diagnostics of malignant diseases based on the pathologic changes in the tissues (47). PET/CT is with a high sensitivity and specificity in diagnosis and treatment of the colorectal cancer in comparison with other diagnostic methods and it is expected in near future it would play main role in this area $(1,31,46$, 47).

Despite of that the method should be used economically effectively because of the high price. It is useful for diagnostics of the tumor formation, for assessment of the dissemination of the process e.g. staging, it gives prognosis for the development of the disease, for plan of the treatment, for assessment of the therapeutic response and for establishment of the possible development of relapse (48). In a prospective trial is reported that the approach in the treatment of patients with colorectal cancer could be changed in $56 \%$ of the cases in result of PET, because this method diagnoses additional lesions in comparison with conventional imaging which worsens the prognosis (49). These data clearly show the powerful prognostic ability of PET accurately to identify the patients who are believed to be with localized form of the disease in comparison with conventional imaging diagnostics (49). Despite the diagnostics of the colorectal cancer, PET/CT does not provide assessment of the tumor invasion, but provides better information for the status of the distant lymph nodes and the metastases in them $(1,31$, $50)$.

${ }^{18}$ F-2-fluorine-2-desoxi-D-glucose

(FDG) is the most frequently used radiopharmaceutic in oncology at the moment. The semi-quantitative analysis of FDG-PET images is performed by calculation of the value of the standard uptake value (SUV). Falsely positive results could be obtained in existing chronic inflammation and after operation or radiotherapy, due to secondary increased uptake of FDG in neutrophils, granulation tissue and macrophages (46). Commonly accepted is the role of FDG-PET in the assessment of the therapeutic effect after radiotherapy, chemotherapy and after usage of ablative technics $(46,51)$. The examination could be performed 4 weeks after termination of chemotherapy, one month after operation and 3 months after radiation, because of the possibility for incorrect positive results in inflammatory or regenerative processes. When it is performed in appropriate time after treatment it has potential to offer information for the vitality of the residual tumor mass, to differentiate tumor from fibrosis and to help in prognosis for survival. The method represents the strongest evidence at the moment in assessment of the possibility for relapse and in the selection of patients for metastasis-ectomy. FDG-PET clearly shows the response of the patient to the treatment, especially by finding the metastatic or possibly relapsing process $(1,46,48)$. The diagnostic value for malignant disease from PET is accepted definitely in solid pulmonary nodes $>1 \mathrm{~cm}$ in diameter and SUV > 3. Despite that this diagnostic method could not define if the lesion is primary or secondary (27). In nodes less from $1 \mathrm{~cm}$ in diameter the identification of metastatic nodes by PET is with low sensitivity around $30 \%$ $(33,27)$. Regarding the pulmonary nodes with dimensions in the range 5 to $10 \mathrm{~mm}$, the increased uptake of the isotope increases the probability to register metastatic disease despite that the negative result from PET does not exclude it definitely. In patients with small pulmonary nodes, in which uptake has not been established or it has been minimal on PET images, metastases are found in 20\% (41). In these cases only the histologic examination could give definite answer or the CT follow-up 
of the lesion (41). Biopsy is not recommended because of the particularly high risk for complications after TTNB and eventual possibility for dissemination $(27,41)$.

Based on data from A. Visioni and J. Kim (47) in up to $50 \%$ from unidentified pulmonary nodes at the end are established to be metastases. In these cases PET/CT has sensitivity and specificity $96 \%$ and $83 \%$ respectively. PPV and NPV have been $84 \%$ and 96\%, respectively (47). The authors recommend the usage of PET/CT in noninvasive diagnostics of unidentified pulmonary lesions and focus on the fact that NPV is significantly higher over $90 \%$, which could significantly reduce the need of invasive diagnostic procedures in part of the patients (47). The sensitivity and specificity of PET/CT in diagnostics of colorectal carcinoma is respectively between $89-95 \%$ and $83-92 \%$ (47). According to another study the sensitivity, specificity and accuracy in finding of unexpected damages from the method are $100 \%, 97,9 \%$ and $98,3 \%$ respectively (52). The data from S. Hahn et al. (53), the sensitivity for automatic finding by PET/CT of colorectal hepatic metastases is $96 \%$ (86-99\%) and $90 \%$ (70-99\%) for pulmonary lesions. PPV is $80 \%$ for the hepatic and $68 \%$ for pulmonary lesions with median values of SUVmax 8,8 for the first and 9,3 for the second, respectively.

PET improves capacity of the computed tomography in diagnosing and assessment of pulmonary metastases (42). In retrospective analysis, performed by Reinherdt et al. about sensitivity of PET for lesions with dimensions from 11 to $29 \mathrm{~mm}$, is established that it reaches the highest values to $94 \%$ and is around $78 \%$ for metastases with dimensions between 8 and $10 \mathrm{~mm}$. Despite that for alterations with size from 5 to $7 \mathrm{~mm}$ in the biggest diameter, the sensitivity is only $41 \%$, which shows the limitations of the method for findings with these or smaller dimensions (42). The simultaneous usage of the abilities of PET/CT could offer better accuracy of almost $99 \%$ for the bigger nodes (42).

Performing a literature survey about the abilities of PET/CT in comparison with PET, C.D. Collins (48) reports that the accuracy in defining the diagnosis in the different malignant diseases is from $90 \%$ to $98 \%$ with reduction of $50 \%$ in the unclear cases. In suspicion of oncology disease, the image of PET/CT offers additional information in 49\% of the cases. The method demonstrates reduction of the level of uncertainty from $15,3 \%$ to $3,4 \%$ (48). FDG-PET helps to be specified the CT findings in the lymph nodes with unclear origin (48).

The diagnostic abilities of FDG-PET and PET/CT in patients with colorectal cancer are indisputable for the distribution of the oncology disease (staging) and the appearance of local in the liver or in the lungs relapses after operative treatment (48). In cases of inflammatory diseases is possible increased uptake of FDG in the tissues. Despite the reported high sensitivity, lesion $<0.7 \mathrm{~cm}$ could not be established accurately. That is why $\mathrm{PET} / \mathrm{CT}$ is not recommended for routine diagnosis because it is nor efficient nor profitable. If the main tumor mass is bulky, small neighbouring lymph nodes could not be detected as separate structures on the PET image, which is a special advantage of the CT, despite that it is also not able of identifying micro-metastases. MRI is the recommended method for imaging of meso-rectal infiltration (48). Based on literature data comparing the abilities of CT with additional contrasting and $\mathrm{PET} / \mathrm{CT}$, the conclusion is that there is little difference in the sensitivity of the two methods - $95 \%$ and $91 \%$, respectively. In other scientific reports for patients with preceding hepatic surgery this difference is significant $100 \%$ versus $50 \%$ in favor of PET/CT. This method has accuracy of $88 \%$ in diagnosis of relapsing colorectal cancer in comparison with $71 \%$ for PET. In additional study PET/CT demonstrates sensitivity $89 \%$, specificity $92 \%$ and accuracy of $90 \%$ for locally relapsing colorectal cancer, the liver and the extraabdominal localizations. The method has also high sensitivity in finding of extra-hepatic distribution of $89 \%$ in comparison with $64 \%$ for $\mathrm{CT}$ and leads to change in therapeutic strategy in $21 \%$ of the cases (48).

PET provides information for inclusion of the mediastinal lymph nodes and for finding of extra-pulmonary progression of the disease (33). But according to other studies the sensitivity of PET/CT for finding metastases in mediastinal lymph collectors is only 35\% (54). According to the data by K.M. Musallam et al. (4), ${ }^{18}$ FDG-PET scanning is proved superior to the morphologic imaging diagnostics in the assessment of the participation of lymph node based on data for functional assessment of the tumor metabolism. The analysis of the contemporary scientific literature establish that the change of therapeutic plan based on 18FDG PET scan of the patients with metastatic colorectal cancer is found in $31.6 \%$ of the cases (4). Despite that small malignant lesions could not show increased uptake of radioactive isotope because the present PET - detectors 
offer spatial resolution ability of only $5 \mathrm{~mm}$. This limitation is strongest in the chest, where finding of lesions with PET is additionally compromised from the breathing moves. Additionally, extensive fibrosis of mediastinal lymph nodes could lead to decrease of uptake of 18F-FDG during the initial PET scanning. Exceptional results are reported about the tumor staging when using PET/CT (4). Numerous studies show that FDG-PET has high sensitivity and high specificity for finding of tumor relapse in patients with colorectal carcinoma and increasing levels of CEA, in which there are no unidentified suspicious findings by the standard imaging techniques. The combination PET/CT outweighs CT and MRI for finding of local relapses and metastatic progression in the liver and other distant places. Despite that the current systems for scanning have some limitations:

1. One of the most important is its low sensitivity for finding of small lesions between 5 and $10 \mathrm{~mm}$.

2. The difficult assessment about the invasion of the local tumor in the surrounding tissues.

3. 18F-FDG is limited from the fact that it is not specific for tumors but the uptake of the radio-pharmaceutic could happen in benignant tissues like for example infection or inflammation (55).

In conclusion we can state that FDG-PET and PET/CT play significant role for defining the accurate diagnosis of the main disease, the staging and finding of relapse occurrence after treatment. There is more and more evidence that these methods could contribute significantly for assessment of the response from the therapy and for the planning of the treatment $(56,57)$.

Bronchoscopy - The endobronchial metastases from colorectal cancer are rare (58). These could be suspected when they appear after the fifth year (58) and often they are related with increase of the CEA levels without other focal manifestations. Bronchoscopy is not usually in the armory of the colorectal surgeon, but in the conditions of lobar atelectasis it is especially useful method for direct view and histologic verification $(14,26$, $59,60)$. While $95 \%$ of the pulmonary metastases are detectable on CT, only $55 \%$ of the endobronchial lesions are defined by this method. (26). The damages are registered in segment bronchia in $56 \%$, in lobar bronchia in $33 \%$, in main bronchia in $6 \%$, and in $5 \%$ of the cases they are found in different places (25). In nearly $72 \%$ of the patients the metastases have been localized in the right part of the bronchial tree (25). The bronchoscopy is important to prove the origin of the tumor formation (endobronchial metastasis or primary cancer of the lung) (59).

According to S. Tanaka et al. (61), despite the distinctive signs of the metastatic colorectal adenocarcinoma from primary adenocarcinoma of the lungs, often the distinction is difficult, but identification is very important for the surgery treatment, because the dimensions of the resection for every diagnosis differ significantly. The examination of CDX2 gene is specific marker for defining the probability of development of colorectal cancer as all of the samples should undergo immunehistochemical coloring for CDX2, citokeratine (CK)7, CK20 and thyroid transcription factor (TTF -1). Positive CDX2 coloring represents a highly sensitive and specific marker of the metastatic colorectal carcinoma, in comparison with the other tests which have lower diagnostic value (61). In some patients TTNB under echography control before the metastase-ectomy is indicated for exclusion of metastases in the mediastinal lymph nodes. Their proving makes the surgical treatment pointless because of the bad prognosis (62).

Mediastinoscopy - This method is used when suspecting and for proving of metastases in mediastinal lymph nodes, because in $14 \%$ to $42 \%$ of the patients these are established $(14,63)$. Sensitivity of CT in these cases is only $73 \%$ (63).

Videothoracoscopy (VATS) - for identification of lymph metastases in defined group mediastinal lymph nodes and superficial suspicious nodes in the lungs.

Trans-thoracic needle biopsy (TTNB) - for wall-attached unidentified pulmonary findings, but with diameter bigger than $3 \mathrm{~cm}$.

Tumor markers - CARCINOEMBRIONIC ANTIGEN (CEA) - The level of CEA in serum before thoracotomy is one of the most significant prognostic factors in univariate and multivariate analysis for survival after resection of metastatic tumors of the lungs (15, $17,50,64,65)$. As a whole the increased serum level of CEA is related with the biologic functions of the colorectal carcinoma because its increased expression correlates with bad prognosis not only with organ localization but with its metastatic progression in the lungs (50, 64). The size of the tumor, the number of the pulmonary lesions and the metastases in the lymph nodes represent the extent of metastatic pulmonary disease and they appear as prognostic factors similar to these included in TNM classifications $(15,66)$. In support of the 
latter thesis are the data of the 5 year survival rate of patients with normal or increased levels of CEA, respectively $51,9 \%$ for the first versus $38,3 \%$ for the second group of patients (29). This difference is significant in the publication of P.J.Villeneuve and R. S. Sundaresan (5), where it is $60 \%$ for the first indicator versus $18 \%$ for the second group of patients with increase of CEA over $10 \mathrm{ng} / \mathrm{ml}$. Similar are the data cited by R. Maeda et al. (67) based on the same criteria but with significantly higher values $91,7 \%$ versus $42,6 \%$ respectively. $\mathrm{T}$. Treasure et al. (68) report that they have established increased levels of CEA over 5 $\mathrm{ng} / \mathrm{ml}$ in $3 \%, 25 \%, 45 \%$ and $65 \%$ of the patients in accordance with the stages they have been classified by Dukes A, B, C and D, respectively. After radical resection the serum concentration of CEA drops. According to numerous publications increase of CEA is related with bad outcome after pulmonary metastases-ectomy of the patients and it could be accepted as a contraindication for pulmonary resection $(52,68,69,70)$. On the contrary, J.H. Kim et al. (71) support the standpoint that increased serum levels of CEA should not be taken as contraindication for surgical treatment because the survival after pulmonary metastases-ectomy in patients with normal and high levels are almost the same. Similar is the opinion of S. Limmer et al. (72), who consider that the increased level of CEA and the number of lesions could influence over survival but are not absolute contraindication for surgical resection.

It is supposed that the high values of CEA could be one of the most important indicators for progression of colorectal adenocarcinoma (17). The serum concentration of the antigen is usually considered as an indicator for the volume of the tumor (57). It is well-known that CEA participates in the cell structure and encourages the adhesion of the tumor cells to the normal of the organism. Consequently, its level could be a reflection of the malignancy of the tumor (73). According to R. Koga et al. (73), the time needed for the occurrence of metastases and the level of carcinoembryonic antigen before the thoracotomy could have decisive meaning for the prognosis of the surgical treatment. T. Irvine, M. Scott and C. Clark (74), report that increase of CEA with 1 $\mathrm{ng} / \mathrm{ml}$ correlates with relapse of the metastases. In $33 \%$ of the patients with these changes in the final analysis a relapse of the disease is established. Increase of CEA with 1 unit has predictive value of $74 \%$ for relapse or metastases (sensitivity $80 \%$, specificity $86 \%$ ). Previous studies point the role of the significant increase in the serum levels of CEA at the occurrence of relapse, but this shows that small changes in CEA could be significant even if these levels are in the range of "normal" limits (74).

According to P.C. Lin et al. (75), the high level of CA19-9 remains independent prognostic factor for patients with normal levels of CEA. The patients with high level of CA19-9 also show more frequent metastases in the lungs in comparison with these with normal levels, $23,1 \%$ versus $7,2 \%$ respectively (75).

\section{CONCLUSION}

The colorectal cancer keeps first place as an etiologic reason for performance of pulmonary metastases resection $(76,77)$. It is established the low effectiveness of the chemotherapy in metastatic progress of the colorectal carcinoma. Subsequently the surgical resection aiming the removal of pulmonary metastases is commonly accepted as the only healing therapeutic approach. In favor of the operative treatment is the trend of earlier finding of the pulmonary metastases. The accurate diagnostics contributes for this.

\section{REFERENCES}

1. Takahashi W., Nakajima M., Yamamoto N. et al. Carbon ion radiotherapy foro ligorecurent lung metastases from colorrectal cancer: a feasibility study. Radiat Oncol 2014: 1: 9: 1: 68.

2. Huang C.W., Tsai H.L., ChenY.T. et al. The prognostic values of EGFR expression and KRAS mutation in patients with synchronous or metachronous metastatic colorectal cancer. BMC Cancer 2013; 13 : 599. Published online Dec 13, 2013. doi 10.1186/1471-2407-13-599.

3. Schweiger T., Hegedüs B., Nikolowski C. et al. EGFR, BRAF and KRAS Status in Patients Undergoing Pulmonary Metastasectomy from Primary Colorectal Carcinoma: A Prospective Follow-Up Study. Ann Surg Oncol 2014: 21:3:946-54.

4. Mussallam K.M., Taher A.T., Tawil A.N. et al. Solitary mediastinal lymph node metastasis in rectosigmoid carcinoma: a case report. Cases J 2008: 31:1:1:69.

5. Villeneuve P.J. Sundraresan R.S. Surgical management of colorectal lung metastasis. Clin Colon Rectal Surg. 2009; 22: 4: $233-$ 41.

6. Meulenbeld H.J., Creemers G.J. First-line treatment strategies for elderly patients with metastatic colorectal cancer. Drugs Aging 2007: 24:3: 223-38.

7. Valerianova Z., Dimitrova N., Tonev S., Vukov M.(eds.) Cancer Incidence in Bulgaria, 2012. Bulgarian National Cancer Registry. Sofia, 2014. 
8. Shah A., Alberts S., Adam R. Accomplishments in 2007 in the management of curable metastatic colorectal cancer. Gastrointest Cancer Res. 2008: 2: 3: Suppl:S13-8.

9. Tampellini M., Ottone A., Bellini E. et al. The role of lung metastasis resection in improving outcome of colorectal cancer patients: results from a large retrospective study. Oncologist 2012: 17: 11: 1430-8.

10.Grossman I., Avenarius J.K., Mastboom W.J. et al. Preoperative staging with chest $\mathrm{CT}$ in patients with colorectal carcinoma: not as a routine procedure. Ann Surg Oncol. 2010: 17: 8: 2045-50.

11.Szöke T., Kortner A., Neu R. et al. Is the mediastinal lymphadenectomy during pulmonary metastasectomy of colorectal cancer necessary? Interact Cardiovasc Thorac Surg. 2010: 10: 5: 694-8.

12.Mongil Poce R., Pagés Navarrete C., Ruiz Navarrete J.A. et al. Survival analysis of resection of lung metastases from colorectal cancer. Arch Bronconeumol. 2009: 45: 5: 235-9.

13.Schneider R., Leinung S., Klöppel R. et al. The preoperative multislice spiral CT in the surgical management of pulmonary metastases. Zentralbl Chir. 2008: 133: 6: 568-73.

14.Dahabre J., Vasilaki M., Statopoulos G.P. et al. Surgical management in lung metastases from colorectal cancer. Anticancer Res. 2007: 27: 6C: 4387-90.

15.Iizasa T., Suzuki M., Yoshida S. et al. Prediction of prognosis and surgical indications for pulmonary metastasectomy from colorectal cancer. Ann Thorac Surg. 2006: 82: 1: 254-60.

16.Onaitis M.W., Petersen R.P., Haney J.C. et al. Prognostic factors for recurrence after pulmonary resection of colorectal cancer metastases. Ann Thor Surg. 2009:87:6:1684-8.

17.Rama N., Monteiro A., Bernardo J.E. et al. Lung metastases from colorectal cancer: surgical resection and prognostic factors. Eur J Cardiothorac Surg. 2009: 35:3:444-9.

18. Treasure T., Milosevic M., Fiorentino F., Macbeth F. Pulmonary metastasectomy: what is the practice and where is the evidence for effectivness? Thorax 2014: Jan 9. [Epub ahead of print]

19.Zisis C., Tsakiridis K., Kougioumtzi I. et al. The management of the advanced colorectal cancer: management of the pulmonary metastases. J Thorac Dis. 2013: 5: Suppl 4:S383-S388.

20.Warwick R., Page R. Resection of pulmonary metastases from colorectal carcinoma. Eur J Surg Oncol. 2007: 33:Suppl 2:S59-63.

21.Jegatheeswaran S., Satyadas T., Sheen A.J. et al. Thoracic surgical management of colorectal lung metastases: a questionnaire survey of members of the Society for Cardiothoracic Surgery in Great Britain and Ireland. Ann $R$ Coll Surg Engl. 2013: 95:2:140-3.

22.Gielen C., Sanli I., Stroeken L. et al. Staging chest radiography is not useful in patients with colorectal cancer. Eur J Surg Oncol. 2009: 35:11:1174-8.

23.Lee J.H., Ahn B.K., Nam Y.S., Lee K.H. Clinical usefulness of preoperative and postoperative chest computed tomography for colorectal cancer. $J$ Korean Soc Coloproctol. 2010: 26:5:359-64.

24.Christoffersen M.W. Bulut O., Jess P. The diagnostic value of indeterminate lung lesions on staging chest computed tomographies in patients with colorectal cancer. Dan Med Bull. 2010: 57:1:A4093.

25.Kim J.H., Min D., Song S.H. et al. Endobronchial metastases from extrathoracic malignancies: recent 10 years' experience in a single university hospital. Tuberc Respir Dis (Seoul). 2013: 74:4:16976.

26.Fournel C., Bertoletti L., Nguen B., Vergon J.M. Endobronchial metastases from colorectal cancers: natural history and role of interventional bronchoscopy. Respiration 2009:77:1:63-9.

27.Varol Y., Varol U., Karaca B. et al. The frequency and significance of radiologically detected indeterminate pulmonary nodules in patients with colorectal cancer. Med Princ Pract. 2012:21:5:457-61.

28.Nordhom-Carstensen A., Krarup P.M., Jorgensen L.N. et al. Occurrence and survival of synchronous pulmonary metastases in colorectal cancer: a nationwide cohort study. Eur $J$ Cancer. 2014: 50:2:447-56.

29. Inoue M, Ohta M, Iuchi K, Matsumura A, Ideguchi K, Yasumitsu T, Nakagawa K, Fukuhara K, Maeda H, Takeda S, Minami M, Ohno Y, Matsuda H; Thoracic Surgery Study Group of Osaka University. Ann Thorac Surg. 2004 Jul;78(1):238-44.

30.Renaud S., Alifano M., Falcoz P.E. et al. Does nodal status influence survival? Results of a 19-year systematic lymphadenectomy experience during lung metastasectomy of colorectal cancer. Interact Cardiovasc Thorac Surg. 2014:18:4:482-7.

31.Pfannschmidt J., Egerer G., Bischof M. et al. Surgical Intervention for Pulmonary 
Metastases. Dtsch Arztebl Int. 2012:109:40:645-51.

32.Jess P., Seiersen M., Ovesen H. et al. Has PET/CT a role in the characterization of indeterminate lung lesions on staging CT in colorectal cancer? A prospective study. Eur J Surg Oncol. 2014: 40: 6: 719-22.

33.Zabaleta J., Aguinagalde B., Izuierdo J.M. et al. Determination of a low risk group for having metastatic nodules not detected by computed tomography scan in lung metastases surgery. Arsh Bronconeumol. 2013: 49:12:518-22.

34.Kawaguchi T., Kusumoto M., Maeshima A. et al. High-resolution computed tomography appearances of surgically resected pulmonary metastases from colorectal cancer, with histopathologic correlation. Radiat Med. 2005: 23:6:418-26.

35.Scott A.M., Gunaawardana D.H., Kelley B. et al. PET changes management and improves prognostic stratification in patients with recurrent colorectal cancer: results of a multicenter prospective study. $J$ Nucl Med. 2008: 49:9:1451-7.

36.Lin B.R., Chang T.C., Lee Y.C. et al. Pulmonary resection for colorectal cancer metastases: duration between cancer onset and lung metastasis as an important prognostic factor. Ann Surg Oncol. 2009: 16:4:1026-32.

37.Nordholm-Carstersen A., Wille-Jørgensen P.A., Jorgensen L.N., HarlingH. Indeterminate pulmonary nodules at colorectal cancer staging: a systematic review of predictive parameters for malignancy. Ann Surg Oncol. 2013: 20:12:4022-30.

38. Brent A., Talbot R., Coyne J. Nash G. Should indeterminate lung lesions reported on staging CT scans influence the management of patients with colorectal cancer? Colorectal Dis. 2007: 9:9:816-8.

39. Nakajima J., Murakawa T., Fukami T. et al. Is finger palpation at operation indispensable for pulmonary metastasectomy in colorectal cancer? Ann Thorac Surg. 2007: 84:5:1680-4.

40.Quyn A.J., Matthews A., Daniel T. et al. The clinical significance of radiologically detected indeterminate pulmonary nodules in colorectal cancer. Colorectal Dis. 2012: 14:7:828-31.

41.Gomez D., Kamali D., Dunn W.K. et al. Outcomes in patients with indeterminate pulmonary nodules undergoing resection for colorectal liver metastases. $H P B$ (Oxford) 2012:14:7:448-54.

42.Timmernnan R.D., Bizekis C.S., Pass H.I. et al. Local surgical, ablative, and radiation treatment of metastases. CA Cancer J Clin. 2009: 59:3:145-70.

43. Marron Fernandez C., de la Cruz J., Lora D. et al. Comparison of pulmonary nodule detection rates between computed tomography, intraoperative lung palpation, and pathologic analysis in patients who undergo pulmonary metastasectomy in colorectal cancer. Chest. 2014: 1:145(3 Suppl).

44.Dong S. Zhang L., Li W. et al. Evaluation of video-assisted thoracoscopic surgery for pulmonary metastases: a meta-analysis. PLoS One 2014: 9;9(1):e85329.

45.Georgieva N., Obretenov E., Kalaydjiev G., Peeva K. Lung Perfusion Scintigrphy in PulmonaryMetastases. Trakia Journal of Sciences 2014:12: Suppl 1:290-294.

46.Herbertson R.A., Lee S.T., Tebbutt N., ScottA.M. The expanding role of PET technology in the management of patients with colorectal cancer. Ann Oncol. 2007: 18:11:1774-81.

47. Visioni A., Kim J. Positron emission tomography for benign and malignant disease. Surg Clin North Am. 2011: 91:1:249-66.

48.Collins C.D. PET/CT in oncology: for which tumours is it the reference standard? Cancer Imaging. 2007: Oct 1;7 Spec No A:S77-87.

49.ScottA.M., Gunawardana D.H., Kelley B. et al. PET changes management and improves prognostic stratification in patients with recurrent colorectal cancer: results of a multicenter prospective study. $J$ Nucl Med. 2008: 49:9:1451-7.

50.Embún R., Fiorentino F., Treasure T. et al. Pulmonary metastasectomy in colorectal cancer: a prospective study of demography and clinical characteristics of 543 patients in the Spanish colorectal metastasectomy registry. BMJ Open 2013 May 28; 3(5). pii: e002787.

51.Krause B.J., Schwarzenböck S., Souvatzoglou M. FDG PET and PET/CT. Recent Rezults Cancer Res. 2013:187:35169.

52.Kodama H., Yamakado K., Takaki H. et al. Impact of 18F-FDG-PET/CT on treatment strategy in colorectal cancer lung metastasis before lung radiofrequency ablation. $\mathrm{Nucl}$ Med Commun. 2013: 34:7:689-93.

53.Hahn S., Heusner T., Zhou X. et al. Computer-aided detection (CAD) and assessment of malignant lesions in the liver and lung using a novel PET/CT software tool: initial results. Rofo 2010: 182:3:243-7.

54.Hamaji M., Cassivi S.D., Shen K.R et al. Is lymph node dissection required in 
pulmonary metastasectomy for colorectal adenocarcinoma? Ann Thorac Surg. 2012: 94:6:1796-800.

55.Sarikaya I., Povoski S.P., Al-Saif O.H. et al. Combined use of preoperative 18F FDGPET imaging and intraoperative gamma probe detection for accurate assessment of tumor recurrence in patients with colorectal cancer.World J Surg Oncol. 2007:16:5:80.

56.Krause B.J., Schwarzenböck S., Souvatzoglou M. FDG PET and PET/CT. Recent Rezults Cancer Res. 2013:187:35169.

57.Muñoz Llarena A., CarreraRevila S., GilNegrete Laborda A. et al. Prognostic factors associated with resectable pulmonary metastases from colorectal cancer. Arch Bronconeumol. 2007:43:6:309-16.

58. Ghetie C., Davies M., Comfeld D. et al. Expectoration of a lung metastasis in a patient with colorectal carcinoma. Clin Colorectal Cancer. 2008: 7:4:283-6.

59.Kim A.W., Liptay M.J., Saclarides T.J., Warren W.H. Endobronchial colorectal metastasis versus primary lung cancer: a tale of two sleeve right upper lobectomies. Interact Cardiovasc Thorac Surg. 2009: 9:2:379-81.

60.Bar-Gil Shirit A., Shirit D., Bakal I. et al. Endobronchial metastases from colon cancer without liver metastases: report of eight cases. Dis Colon Rectum. 2007: 50:7:1087-9.

61.Tanaka S., Saito K., Ito T. et al. CDX2 as a useful marker of colorectal adenocarcinoma metastases to lung in pre-operative biopsy specimens. Oncol Rep. 2007: 18:1:87-92.

62.Eckardt J., Licht P.B. Thoracoscopic versus open pulmonary metastasectomy: a prospective, sequentially controlled study. Chest. 2012: 142:6:1598-602.

63.Menon A., Milton R., Thorpe J.A. Papagiannopoulos K. The value of videoassisted mediastinoscopy in pulmonary metastasectomy. Eur J Cardiovasc Surg. 2007: 32:2:351-4.

64.Vodicka J., Spidlen V., Simánec V., Safránek J. et al. Surgical therapy of pulmonary metastases of colorectal cancer-ten-year results. Rozhl Chir. 2012: 91:2:816.

65.Park J.S., Kim H.K., Choi Y.S. et al. Outcomes after repeated resection for recurrent pulmonary metastases from colorectal cancer. Ann Oncol. 2010: 21:6:1285-9.

66. Hattori N., Kanemitsu Y., Komori K. et al. Outcomes after hepatic and pulmonary
OBRETENOV E., et al.

metastasectomies compared with pulmonary metastasectomy alone in patients with colorectal cancer metastasis to liver and lungs. World J Surg. 2013: 37:6:1315-21.

67. Maeda R., Isowa N., Onuma H. et al. Pulmonary resection for metastases from colorectal carcinoma. Interact CardioVasc Thorac Surg 2009;9:640-644.

68.Treasure T., Milosevic M., Fiorentino F., Macbeth F. Pulmoary metastasectomy: what is the practice and where is the evidence for effectiveness? Thorax. 2014: Jan 9. [Epub ahead of print].

69. Malaisé M., Rovira J., Renner P. et al. KIRG1+ NK Cells Protect T-bet-Deficient Mise from Pulmonary Metastatic Colorectal Carcinoma. J Immunol. 2014:15:192:4:1954-61.

70.Otani Y., Shomura H., Aiyama T. et al. [Prognostic factors affecting the surgical treatment of metastatic lung tumor from colorectal cancer]. K Kyobu Geka 2013: 66:4:279-83.

71.Kim H.J., Kye B.H., Lee J.I. et al. Surgical resection for lung metastases from colorectal cancer. $J$ Korean Soc Coloproctol. 2010: 26:5:354-8.

72.Limmer S., Oevermann E., Killaitis C. et al. Sequential surgical resection of hepatic and pulmonary metastases from colorectal cancer. Langbecks Arch Surg 2010:395:8:1129-38.

73.Koga R., YamamotoJ., Saiura A. et al. Surgical resection of pulmonary metastases from colorectal cancer: Four favourable prognostic factors. Jpn J Clin Oncol. 2006: 36:10:643-8.

74.Irvine T., Scott M., Clark C.I. A small rise in CEA is sensitive for recurrence after surgery for colorectal cancer. Colorectal Dis. 2007: 9:6:527-31.

75.Lin P.C., Lin J.K., Lin C.C. et al. Carbohydrate antigen 19-9 is a valuable prognostic factor in colorectal cancer patients with normal levels of carcinoembryonic antigen and may help predict lung metastasis. Int $J$ Colorectal Dis. 2012: 27:10:1333-8.

76.Poletti G.B., Toro I.F., Alves T.F. et al. Descriptive analysis of and overall survival after surgical treatment of lung metastases. J Bras Pneumol 2013: 39:6:650-8.

77.Petrov D., Stanoev V., Vukov M. et al. Surgical Treatment of Pulmonary Metastases - Surgical Methods, Early and Long-Term Results. Surgery. 2008: LXIV:3.(in Bulgarian). 